\title{
Revisión
}

\section{Asesoramiento genético a mujeres en edad fértil sobrevivientes de cáncer}

\author{
Isabel Castro-Volio
}

\section{Resumen}

Las personas que han padecido cáncer en su infancia, adolescencia, o antes de la menopausia, y han sobrevivido, merecen, además de cantidad, calidad de vida. Un aspecto muy importante del bienestar personal se relaciona con la procreación. Lamentablemente, es posible que las mismas terapias que les han salvado la vida, puedan comprometer su fertilidad, el pronóstico del embarazo y del parto, o la salud del bebé. De igual manera, en aquellos tipos de cáncer con influencia hormonal, nace la duda sobre la prudencia de un embarazo posterior. Otro aspecto fundamental es la posibilidad de transmitir el cáncer a generaciones futuras. El objetivo de esta revisión es apoyar al personal de salud en el abordaje de esta temática e ilustrar la forma como el asesoramiento genético puede contribuir al manejo de estos casos.

Descriptores: cáncer, embarazo, asesoramiento genético, sobrevivientes.

Key words: cancer, pregnancy, genetic counseling, survivors.

Recibido: 13 de marzo de 2007

Aceptado: 31 de julio de 2007

Durante los últimos 30 años se ha producido un dramático incremento en las tasas de sobrevivencia de las personas afectadas de cáncer, gracias a nuevas y mejores terapias. La mayoría de las niñas, adolescentes y mujeres jóvenes con cáncer vivirán muchos años después del diagnóstico, lo cual significa que estas personas, sus familias y terapeutas, deberán discutir temas relacionados con la calidad de vida que tendrán, y entre ellos, la reproducción futura ocupa un lugar muy importante. En este sentido, los aspectos por tratar son básicamente los relacionados con las secuelas sobre la reproducción que normalmente acarrean los mismos tratamientos que les han salvado la vida. ¿La cirugía, la radioterapia o la quimioterapia pueden perturbar la fertilidad, la salud del producto de la concepción o la lactancia? ¿El embarazo puede comprometer la sobrevivencia de la gestante? Trataremos de responder a estas interrogantes a la luz del conocimiento científico actual y desde el punto de vista del asesoramiento genético. También nos

Sección de Genética Humana. Instituto de Investigaciones en Salud (INISA). Universidad de Costa Rica

Abreviaturas:ASCO,Sociedad Americana de Oncología Clínica; CCSG, estudio de sobrevivencia del cáncer infantil; OR, odds ratio.

Correspondencia: Isabel Castro Volio. INISA, Universidad de Costa Rica

Correo electrónico: icastro@ cariari.ucr.ac.cr

ISSN 0001-6002/2007/49/4/190-197 Acta Médica Costarricense, $\mathbb{0} 2007$ Colegio de Médicos y Cirujanos ocuparemos brevemente del cáncer de tipo hereditario, en particular del cáncer de mama, puesto que los factores reproductivos son fuertes modificadores del riesgo de padecerlo, entre las portadoras de mutaciones que confieren predisposición al este.

\section{Asesoramiento genético, conceptos básicos:}

El asesoramiento genético se define como un proceso de comunicación que se ocupa de los problemas humanos asociados con la ocurrencia o riesgo de ocurrencia de un trastorno genético en una familia. Este proceso implica el intento, por parte de una o más personas apropiadamente entrenadas, de ayudar al individuo o a la familia a: a) comprender los aspectos médicos, incluyendo el diagnóstico, curso probable de la enfermedad, y manejo disponible; b) apreciar la forma como la herencia contribuye al padecimiento y el riesgo de recurrencia en parientes específicos; c) entender las opciones para enfrentarse con los riesgos de recurrencia; d) escoger el camino que parece más apropiado para ellos, tomando en cuenta su riesgo, las metas familiares 
y sus principios éticos y religiosos, y actuar de acuerdo con tal decisión, y e) hacer que la familia o el miembro afectado se adapten de la mejor manera al padecimiento y a su riesgo de recurrencia.

¿Qué sucede en una consulta genética? Lo primero es recolectar información; es necesario explorar con el paciente y la familia el motivo de referencia, sus conocimientos de genética, el diagnóstico en consideración, la percepción de estatus de la enfermedad o del riesgo, las creencias acerca de la causa de la enfermedad, la percepción de la carga que esta produce, el historial médico desde el nacimiento hasta el presente, y la historia familiar en primer orden (padres, hijos y hermanos), en segundo orden (abuelos, nietos, tíos, sobrinos y medio hermanos) y en tercer orden (primos hermanos). Para hacer esto se cuenta con una herramienta valiosa llamada el pedigrí, que es el diagrama que registra la información sobre la historia familiar y sirve para convertir los datos proporcionados por el paciente y obtenidos del expediente médico, en un formato estandarizado. En él se demuestran las relaciones biológicas del paciente con los miembros de su familia mediante el uso de símbolos, líneas verticales y horizontales y abreviaturas. Al completarse, se convierte en un registro visual rápido y confiable que ayuda al asesoramiento genético. El análisis del pedigrí puede revelar el patrón de herencia, la segregación, de una enfermedad en una familia y ofrece un medio para estimar el riesgo de recurrencia de la enfermedad en la descendencia.

En caso de embarazo, además se debe recolectar información sobre el bagaje étnico de la familia, es decir, la presencia de consanguinidad, y es preciso obtener y revisar registros médicos adicionales, incluyendo ensayos diagnósticos, en la embarazada y en familiares afectados, y revisar también la historia social de la familia (educación, empleo y funcionamiento social). Conviene tomar en cuenta las posibles fuentes de apoyo psicosocial (comunal, religiosa, familiar), los conflictos éticos potenciales (confidencialidad, discriminación, falsa paternidad) y la posibilidad de realizar exámenes físicos de la paciente y de otros familiares.

Una vez recolectados, tiene lugar el proceso de evaluación de todos estos datos, lo que implica interpretar el cúmulo de información, consultar referencias relevantes, comparar hallazgos con el diagnóstico de referencia y, finalmente, discutir la impresión diagnóstica. Una vez que el diagnóstico está claro, empieza la labor de asesoramiento. En caso contrario, es necesario hacer el diagnóstico diferencial con otras patologías, lo que puede conducir a sugerir más ensayos de laboratorio o evaluaciones. Cuando el diagnóstico resulta desconocido, no queda más que explicar sobre las patologías descartadas y dar seguimiento.

El proceso de comunicación implica brindar información sobre el padecimiento, adecuada al nivel de cada familia y suficientemente detallada, que contemple al menos el curso normal de la enfermedad, el manejo, posibles intervenciones, el tratamiento, la causa genética y el patrón de herencia, los riesgos para la familia en comparación con los de la población general y las diferentes opciones reproductoras. Otra labor no menos importante es brindar apoyo y ayuda en relación con las respuestas emocionales (choque, incredulidad, alivio, miedo, culpa, tristeza, vergüenza, aceptación), valorar apoyo psicosocial profesional, escuchar con empatía, desarrollar estrategias de comunicación con terceras personas que ignoran su riesgo y referir a grupos de apoyo organizados. El seguimiento del caso contempla la comunicación periódica, posibles ensayos diagnósticos, citas de manejo, documentar el caso para el médico de cabecera y para el paciente, alentar a la familia para mantenerse en contacto, sobre todo en situaciones de embarazo, y la disponibilidad del asesor para evacuar cualquier pregunta futura. ${ }^{1-3}$

\section{Potencial reproductivo de las sobrevivientes de cáncer}

Muchos de los tratamientos que permiten que las personas menores que han sufrido de cáncer sobrevivan y lleguen a la edad adulta, comprometen su habilidad de concebir hijos, de llevar los embarazos a término y de parir bebés sanos. Afortunadamente, para estudiar estos y otros temas existe la gran cohorte multicéntrica del Estudio de Sobrevivencia del Cáncer Infantil (CCSG) y otros estudios más pequeños de series de casos clínicos. ${ }^{4}$

Las sobrevivientes de cáncer infantil que retienen la función ovárica después de completar el tratamiento, cuentan con un aumento del riesgo de desarrollar menopausia prematura, es decir, de cesar de menstruar antes de los 40 años. El riesgo se acerca al 30\% para las que fueron tratadas con agentes alquilantes más irradiación abdominal y pélvica. $^{5}$

Se espera que la mayoría de las mujeres tratadas sean fértiles, tomando en cuenta que solo el $6,3 \%$ de las sobrevivientes del CCSG, que tenían más de 18 años, pero se encontraban con no más de 5 años de haber sido diagnosticadas con cáncer, habían sufrido falla ovárica aguda. ${ }^{6}$ Esta patología se define como la pérdida de la función ovárica durante los cinco años posteriores al diagnóstico. La gran mayoría de quienes resultaron infértiles habían recibido irradiación ovárica. Sin embargo, durante los años en que estas mujeres de la cohorte del CCSG fueron tratadas, eran menos frecuentes los esquemas de quimioterapia que usan dosis altas de agentes alquilantes (ciclofosfamida, clorambucil,melfalan, busulfan, carmustina, lomustina, mecloretamina, procarbazina, cisplatino, nitrosoureas) en relación con las dosis actuales, lo que aumentaría las tasas y la extensión del daño ovárico. Otros estudios en Holanda y Dinamarca encontraron un $8 \%$ de falla ovárica, pero otro $23 \%$ de las mujeres mostraban signos de riesgo inminente de menopausia prematura permanente. ${ }^{7}$ Es posible que muchas sobrevivientes experimenten la menopausia alrededor de los 30 años y es aconsejable informar a las mujeres interesadas en tener hijos que sus años fértiles pueden ser muy pocos. La viabilidad de los 
folículos puede ser reducida aún por dosis bajas de radiación a los ovarios, o por la exposición a la quimioterapia, lo cual acorta los años fértiles de las sobrevivientes. ${ }^{8}$

Un estudio reciente de la Sociedad Americana de Oncología Clínica (ASCO) encontró que en las personas con cáncer, los métodos para preservar su fertilidad se usan con poca frecuencia. Por este motivo, ha girado recomendaciones en tal sentido. ${ }^{9}$ Los pacientes deben ser asesorados acerca de la posible infertilidad, tomando en cuenta factores individuales tales como: tipo de neoplasia, edad del paciente, tipo y dosificaciones del tratamiento que deberán recibir y fertilidad previa a este. Los pacientes interesados en conservar su fertilidad deben considerar las diferentes opciones tan pronto como sea posible, en procura de maximizar las posibilidades de tener éxito. Los dos métodos más exitosos son la criopreservación del esperma para los varones y la congelación de embriones para las mujeres. Existen otras opciones, algunas son estándares y otras se encuentran aún en etapa experimental. ${ }^{10,11}$ Las decisiones en esta materia deben ser abordadas conjuntamente por los pacientes, sus padres o representantes legales, profesionales en oncología, especialistas en endocrinología reproductiva y en psicología y trabajo social. En el sitio Web de la ASCO (http://www.asco.org) se presenta información útil para el médico y para los pacientes, o sus padres, enfrentados con el problema de la infertilidad como secuela de la terapia del cáncer.

\section{Embarazo en las sobrevivientes}

Una vez que la sobreviviente de cáncer infantil o premenopáusico logra un embarazo, surgen tres preocupaciones fundamentales para los futuros padres y para el personal de salud que atiende la gestación. El principal motivo de consulta genética se refiere a las posibles consecuencias que el tratamiento recibido por el cáncer tiene sobre la salud del producto. Esta es la duda que suele agobiar más a la pareja. Otra preocupación, sobre todo para el obstetra, se refiere a las secuelas que puedan haber dejado la terapia en el aparato reproductor femenino y su impacto en el curso del embarazo y del parto. El oncólogo, por su parte, también se preocupará por las consecuencias del embarazo y las posibilidades de recurrencia de la neoplasia.

\section{Riesgos para la salud del producto de la concepción}

Los dos tratamientos fundamentales del cáncer, la quimioterapia y la radioterapia, tienen una gran cantidad de efectos adversos en el corto y largo plazos. Se ha demostrado que son mutagénicos para las células somáticas, causan mutaciones en genes y en cromosomas, y con posterioridad, en los sobrevivientes de cáncer pueden aparecer tumores secundarios relacionados con la terapia. También son mutagénicos para las células germinales y el daño a su material genético puede influenciar la fertilización, aumentar la tasa de abortos o causar malformaciones en la prole de quienes antes fueron expuestos a terapias contra neoplasias. ${ }^{12}$
A pesar de que la información recolectada indica que tanto la quimioterapia como la radioterapia son mutagénicos para los gametos femeninos y masculinos en varios estadios de su maduración, esto no se traduce en un incremento de la cromosomopatía o malformaciones congénitas, en cuanto a la población general. El estudio más grande sobre enfermedad genética en la descendencia de los sobrevivientes de cáncer tratados con mutágenos potenciales, no encontró diferencia significativa en la incidencia de todas las enfermedades de origen genético entre la prole de los sujetos con respecto a la de los controles. Los autores concluyen que de haber daño genético inducido, este sería de un nivel tan bajo que no podría haber sido detectado por los análisis estadísticos. ${ }^{13,14}$ En otra publicación acerca de los efectos transgeneracionales de la radioterapia y con base en evaluaciones dosis respuesta, no se encontró riesgo significativo de enfermedades genéticas hereditarias en la descendencia concebida después de la exposición a la radiación. ${ }^{15}$ En una investigación sobre la inestabilidad cromosómica en relación con la radioterapia administrada por cáncer infantil, se hizo análisis cromosómico en la sangre periférica de los sobrevivientes, sus cónyuges y su prole, sin que se encontrara evidencia de inestabilidad persistente en los irradiados ni de que se hubiera transmitido inestabilidad a las células somáticas de la descendencia a través de la línea germinal del padre o de la madre. ${ }^{16}$ Otro estudio reciente para investigar la tasa de mutaciones germinales de ocho loci minisatelíticos en 24 familias danesas, en las cuales el padre o la madre eran sobrevivientes de cáncer en la infancia o en la adolescencia, el cual había sido tratado con radioterapia, tampoco encontró un aumento estadísticamente significativo de la tasa de mutaciones germinales minisatelíticas. ${ }^{17}$ Son varias las razones que pueden explicar esta discrepancia aparente. En primer término, los espermatozoides mutados pueden ser incapaces de fertilizar el ovocito. En segundo lugar, si ocurre la fertilización las mutaciones letales dominantes pueden provocar el aborto inadvertido de un embarazo incipiente. También es posible que el ovocito tenga mecanismos para corregir la cromosomopatía espermática y su propio daño mutacional. La tendencia es hacia una disminución de la frecuencia de anomalías genéticas largo tiempo después de la exposición a agentes quimioterapéuticos. ${ }^{18}$ Con respecto a las mujeres que han recibido altas dosis de radioterapia antes de concebir, y cuya fertilidad no ha sido perturbada, parece que el peligro de que el bienestar del bebé se vea amenazado es muy pequeño. Por el contrario, la exposición a altas dosis de radiación durante el embarazo puede ser letal, teratogénica o carcinogénica para el feto. ${ }^{12}$

\section{Complicaciones obstétricas en las embarazadas que han sobrevivido al cáncer}

Los embarazos en estas mujeres se consideran de alto riesgo obstétrico, generan dudas sobre la ruta óptima del partoyrequierenlaintervencióndeungrupomultidisciplinario para su manejo. ${ }^{19}$ 
En el Instituto Internacional de Epidemiología se investigó si los bebés nacidos de madres tratadas por cáncer infantil tenían un aumento en el riesgo de prematuridad o de padecer restricción de crecimiento fetal, y la asociación de los diferentes esquemas de tratamiento con estas complicaciones del embarazo. ${ }^{20}$ Con datos del CCSG, que comparan a las sobrevivientes con sus hermanas sanas, y tomando en cuenta solo el $65 \%$ de los embarazos que llegaron a término con un recién nacido vivo, se encontró un riesgo moderadamente más alto de prematuridad $(\mathrm{OR}=3,5)$, de bajo peso al nacer $(\mathrm{OR}=6,8)$ y de ser pequeño para la edad gestacional $(\mathrm{OR}=4,0)$. Sin embargo, buena parte de este riesgo se concentra en los bebés cuyas madres fueron irradiadas antes de la menarca y en quienes el útero estaba dentro del campo de radiación. Tales resultados concuerdan con observaciones previas de reducción de la capacidad uterina y del flujo sanguíneo por la radioterapia, especialmente cuando se administra antes de la adolescencia. ${ }^{7}$ Un estudio anterior, también con información del CCSG, también mostró una tendencia hacia tasas aumentadas de abortos $(15 \%)$ y de bajo peso al nacer en los bebés de las mujeres tratadas durante la infancia con irradiación pélvica; en cambio, para quienes recibieron solamente quimioterapia, no se presentaron estos efectos adversos. ${ }^{21}$ Un informe más reciente $^{22}$ determinó que en la historia reproductiva de sobrevivientes holandesas de cáncer infantil, la incidencia de falla ovárica inminente, de abortos y de abortos recurrentes, estaba aumentada.

Otro asunto fundamental, es la posibilidad de que el estrés físico de la gestación pueda desencadenar cardiomiopatía o hipertensión pulmonar de la embarazada, como resultado de daños ocultos producto de la irradiación del pecho o la terapia con antraciclinas. Un estudio parece desestimar el riesgo de desarrollar insuficiencia cardiaca clínica inducida por antraciclina alrededor del momento del parto, ${ }^{23}$ pero es necesario estudiar más profundamente el tema.

\section{Riesgos de recurrencia de neoplasia que puede imponer el embarazo}

En Estados Unidos casi el 25\% de las diagnosticadas con cáncer de mama son mujeres que aún no han enfrentado la menopausia, y muchas de ellas, dada la tendencia actual de posponer la reproducción, tampoco han tenido hijos. A pesar del vínculo entre las hormonas sexuales femeninas y la carcinogénesis mamaria, la posibilidad de que un embarazo posterior al tratamiento curativo por cáncer de mama pueda despertar micrometástasis inactivas, no ha sido demostrada. Por el contrario, parece poco probable que el embarazo tenga un efecto negativo sobre la sobrevivencia de estas mujeres..$^{24,25}$ No hay evidencia de que las pacientes tratadas en estadio temprano para curar el cáncer de mama y que luego quedan embarazadas, comprometan su sobrevivencia, pero deben ser asesoradas tomando en cuenta su riesgo de recurrencia en general. Para quienes tienen un alto riesgo de recurrencia, el embarazo y la maternidad pueden ser una opción imprudente. ${ }^{26} \mathrm{El}$ efecto del embarazo en la sobrevivencia y el mejor momento para concebir aún no están claros, por lo tanto, es esencial tomar en cuenta las prioridades de cada caso, pero en términos generales, se puede recomendar esperar al menos dos años entre el momento del diagnóstico de cáncer de mama y la concepción. ${ }^{27}$ Algunos autores sugieren que para las mujeres con antecedente de cáncer de mama localizado, es poco probable que el embarazo temprano, seis meses después de completar el tratamiento, pueda reducir su sobrevivencia. ${ }^{28}$

Está ampliamente evidenciado que las mujeres sobrevivientes de enfermedad de Hodgkin que fueron tratadas con radioterapia supradiafragmática, tienen aumento del riesgo de desarrollar cáncer de mama. ${ }^{29}$ Según Hill y colaboradores, las mujeres jóvenes tratadas con irradiación al pecho y antraciclinas por causa del linfoma de Hodgkin, tienen un riesgo de desarrollar cáncer de mama que se exacerba con el embarazo. ${ }^{30}$

\section{Embarazo y riesgo de desarrollar cáncer en mujeres con síndromes de cáncer hereditario}

Aunque el cáncer es una enfermedad genética, la mayoría de los casos son esporádicos, sin embargo, algunos tipos han alcanzado una incidencia tan alta, que es posible que en la familia se presente más de un caso, por ejemplo, de cáncer de mama. Para fines del asesoramiento genético, es imprescindible determinar si el caso en consideración pertenece al pequeño grupo de pacientes en quienes es evidente la herencia mendeliana, por lo general autosómica dominante (Cuadro 1). Para distinguir entre cáncer esporádico y cáncer hereditario, se sabe, en general, que los síndromes de cáncer hereditario refieren las siguientes características: a) el mismo tipo de tumor o de tumor secundario o asociado, en dos o más parientes cercanos; b) se presenta en edades más tempranas de lo usual, en uno o más parientes; c) en los órganos pares hay casos de tumor bilateral; d) tumores primarios múltiples en la misma persona; e) se presenta una constelación específica de tumores que son parte de un síndrome conocido, y f) hay evidencia de que la susceptibilidad al cáncer se transmite de manera dominante. ${ }^{3}$ Una herramienta adicional son los ensayos genéticos que permiten salir de dudas y que están disponibles para una variedad de síndromes de cáncer hereditario (Cuadro 2).

El cáncer de mama es una enfermedad muy frecuente en los países occidentales, donde afecta a una mujer de cada 8 ó 15 a lo largo de su vida. El cáncer de mama en nuestro país se ha convertido en un problema de salud pública, ya que según las Normas y Procedimientos de Cáncer en Costa Rica, publicadas en octubre de $2005,{ }^{31}$ ha pasado a ocupar el primer lugar en incidencia. De acuerdo con la base de datos internacional GLOBOCAN $2002^{32}$ y las estadísticas del Ministerio de Salud, se estima en un aproximado de 600-800 los nuevos casos anuales de cáncer de mama en Costa Rica. 
Cuadro 1. Algunos síndromes de cáncer hereditario y sus tumores

\begin{tabular}{|c|c|c|}
\hline Síndrome & Tumor primario & Tumores secundarios o asociados \\
\hline Retinoblastoma genético & Retinoblastoma & Sarcomas, pineoblastoma, melanoma \\
\hline Neurofibromatosis tipo 1 & Neurofibromas & $\begin{array}{l}\text { Neurofibrosarcoma, leucemia mieloide } \\
\text { leucemia mielomonocítica juvenil, glioma }\end{array}$ \\
\hline Neurofibromatosis tipo 2 & Schwanomas vestibulares & Meningiomas, astrocitomas, ependimomas \\
\hline Li - Fraumeni & Sarcomas, cáncer de mama & Tumores adrenocorticales, cerebrales, leucemia \\
\hline Poliposis adenomatosa familiar & Cáncer de colon y rectal & Cáncer del tracto gastrointestinal, hepatoblastoma \\
\hline Gardner & Cáncer de colon y rectal & Hepatoblastoma, tumor desmoide, osteoma \\
\hline Turcot & Cáncer de colon y rectal & Tumores cerebrales, cáncer de tiroides \\
\hline $\begin{array}{l}\text { Cáncer de colon y recto hereditario } \\
\text { no poliposo }\end{array}$ & Cáncer de colon y rectal & Cáncer endometrial, ovárico, gástrico, pancrático \\
\hline Peutz - Jegher & Hamartomas / poliposis & Gastrointestinal, mama, tiroides, tracto genital \\
\hline Enfermedad de Cowden & Hamartomas & Tiroides, colon, mama \\
\hline Cáncer de mama familiar 1 & Cáncer de mama & Ovario, próstata \\
\hline Cáncer de mama familiar 2 & Cáncer de mama & Pancreático, ovárico \\
\hline Neoplasia endócrina múltiple tipo 1 & Cáncer del islote pancreático & Cáncer de paratiroides, de tiroides, de pituitaria \\
\hline Neoplasia endócrina múltiple tipo $2 \mathrm{~A}$ & Tiroides medular & Paratiroides, páncreas, feocromocitoma \\
\hline Neoplasia endócrina múltiple tipo $2 \mathrm{~B}$ & Tiroides medular & Adrenocortical, ganglionar, feocromocitoma \\
\hline Complejo Carney & Adrenocortical & Pituitaria, tiroides, mixomas, testicular, ovárico \\
\hline Beckwith - Wiedemann & Tumor de Wilms & Hepatoblastoma, carcinoma adrenocortical \\
\hline Von Hippel - Lindau & Renal de células claras & Cerebrales, feocromocitoma, \\
\hline Esclerosis tuberosa & Renales & Cerebrales \\
\hline $\begin{array}{l}\text { Carcinoma nevoide de células } \\
\text { basales (Gorlin) }\end{array}$ & Carcinoma de células basales & Méduloblastoma, fibromas, fibrosarcomas \\
\hline Melanoma familiar & Melanoma & Cáncer pancreático \\
\hline Xeroderma pigmentosa* & Cutáneos & \\
\hline Bloom $^{*}$ & Cutáneos & Leucemia linfocítica, linfoma, carcinoma \\
\hline Ataxia - telangiectasia* & Telangiectasia, ataxia & Leucemia linfocítica, linfoma \\
\hline
\end{tabular}

* Estos tres síndromes son de herencia autosómica recesiva y predisponen al paciente a desarrollar neoplasias.

Cuadro 2. Síndromes de cáncer hereditario para los cuales se dispone de ensayos genéticos de uso clínico (*)

\begin{tabular}{|l|l|l|l|}
\hline \multicolumn{1}{|c|}{ Síndrome } & \multicolumn{1}{c|}{ Gen(es)/marcadores } & \multicolumn{1}{c|}{ Herencia } & \multicolumn{1}{c|}{ Ensayo(s) } \\
\hline Cáncer de mama - ovario & BRCA1, BRCA2 & Autosómica dominante & BRCA 1/ BRCA 2 \\
Colon y recto no poliposo & MLH1, MSH2, MSH6, & Autosómica dominante & MLH1, MSH2, MSH6, PreGen-26, Colaris \\
Poliposis adenomatosa & APC & Autosómica dominante & Colaris AP, APC, FAP \\
familiar & TP53 & Autosómica dominante & TP53 \\
Li-Fraumeni & VHL & Autosómica dominante & VHL \\
Von Hippel -Lindau & Autosómica dominante & Melaris \\
Melanoma hereditario & p16 & Autom & \\
\hline
\end{tabular}

* Department of Health and Human Services USA. Agency for Healthcare and Quality. Technology Assessment Program. Genetic Tests for Cancer. Enero 9, 2006. 
Por tal motivo, la discusión se enfoca sobre las repercusiones del embarazo y las posibilidades de transmitir cáncer a las generaciones futuras, particularmente en cuanto al cáncer mamario hereditario o familiar.

El asesoramiento genético en oncología difiere del tradicional que se practica en situaciones prenatales o pediátricas. Se necesita un pedigrí con al menos tres generaciones y documentar todos los diagnósticos de cáncer, con su localización anatómica y las edades en que fue diagnosticado en los miembros de la familia, para identificar el patrón de herencia correcto. Otra información requerida es acerca de los factores de riesgo asociados con estilos de vida y las prácticas de monitoreo del cáncer. El asesoramiento proporciona recomendaciones para la vigilancia del cáncer según el riesgo de cada persona. Estas recomendaciones se realizan de manera dirigida, lo que significa una ruptura con el asesoramiento no dirigido tradicional. ${ }^{33}$ La mayor parte de los casos oncológicos referidos para asesoramiento genético corresponden al cáncer de mama.

La proporción de casos de cáncer de mama que tienen componente hereditario es pequeña, alrededor del $10 \%$. Los principales genes implicados hasta la fecha en este tipo de cáncer son los genes de cáncer de mama - ovario hereditarios 1 y 2, o BRCA1 y BRCA2 y, en menor grado, los genes TP53 (síndrome de Li-Fraumeni), PTEN (síndrome de Cowden), STK11 (síndrome de Peutz-Jeghers), ATM (portadoras de ataxia telangiectasia) y $C H K 2$ (síndrome de cáncer de mama, colon y recto hereditario) ${ }^{33}$. Sin embargo, solo en el $2 \%$ de todos los casos familiares de cáncer de mama, se han identificado mutaciones en los genes BRCA1 y BRCA2, de manera que el defecto genético en la mayoría de los cánceres hereditarios todavía se desconoce, aún más, podría tratarse de un problema de naturaleza poligénica.

En muchas situaciones, el riesgo de desarrollar cáncer de mama de una mujer que tiene historia familiar de esta enfermedad, se puede calcular con base en estudios epidemiológicos, los cuales indican que el riesgo de una mujer que tiene únicamente una pariente en primer orden afectada (madre, hija, hermana), es aproximadamente el doble del riesgo de cualquier mujer de la población general. Los riesgos aumentan si hay más parientes afectadas, o si la pariente afectada fue diagnosticada antes de los 50 años. ${ }^{34}$ Para estimar el riesgo en situaciones más complejas, se pueden usar algoritmos, aunque estos modelos no han sido validados por completo. ${ }^{35}$

En el caso de las familias que han sido estudiadas con ensayos genéticos para identificar mutaciones en los genes de susceptibilidad $B R C A 1$ y $B R C A 2$, el riesgo para las mujeres portadoras de mutaciones es diferente. El riesgo de padecer cáncer de mama en algún momento de su vida para quienes tienen mutaciones en $B R C A 1$ es del $65 \%$ al $85 \%$, y el riesgo para el cáncer de ovario es del 39 al $60 \%$. Para quienes tienen mutaciones en $B R C A 2$, es un poco más bajo, del $45 \%$ al $85 \%$ de riesgo para cáncer mamario y del $11 \%$ al $25 \%$ para el de ovario. ${ }^{33}$ Narod y su grupo ${ }^{36}$ encontraron una frecuencia total de mutaciones BRCA1 y BRCA2 del 13,2\% en la población general de la ciudad canadiense de Ontario. También informaron que la penetrancia de $B R C A 1$ en cuanto a cáncer de ovario fue del $24 \% \mathrm{y}$ del $90 \%$ en el caso de cáncer mamario. La penetrancia de BRCA2 fue del 8,4\% para cáncer de ovario y del $41 \%$ para cáncer de mama. En ambos casos, el riesgo puede ser menor en una familia en la que se ha identificado alguna de estas mutaciones, pero que tiene pocos casos de cáncer entre sus miembros.

En la población general, el aumento en la paridad y la edad de la madre al momento de su primer embarazo de término, son factores de riesgo bien establecidos para el cáncer de mama. Entre los modificadores del riesgo de padecer cáncer de mama hereditario asociado a mutaciones en los genes $B R C A 1$ y $B R C A 2$, los más fuertes son la historia reproductiva, incluyendo la edad de la menarca, la paridad, la lactancia, la ooforectomía y las hormonas exógenas, como los anticonceptivos orales. ${ }^{37}$ No está claro si el efecto de la paridad es diferente según la mutación afecte el gen $B R C A 1$ o el gen $B R C A 2$. Los estudios en portadoras de mutaciones en el gen $B R C A 1$ apuntan hacia una disminución del riesgo de desarrollar cáncer mamario, conforme aumenta la paridad. En un estudio entre las portadoras de mutaciones en BRCA1, la paridad per se no se asoció con el riesgo de cáncer de mama, excepto para las que tienen cuatro o más hijos, en quienes se notó una disminución del riesgo del $38 \%$ en relación con las nulíparas. Por el contrario, entre las portadoras de $B R C A 2$ el aumento en la paridad se asoció con un incremento significativo en el riesgo de padecer cáncer mamario antes de cumplir 50 años, y este riesgo fue mayor en el lapso de dos años después de un embarazo. ${ }^{38}$ En otra investigación, la multiparidad pareció ser protectora en las portadoras de mutaciones en el primer gen, pero se asoció con un aumento del riesgo en las que tuvieron mutaciones en el segundo gen. La ooforectomía se asoció con reducciones en el riesgo de cáncer de mama y de ovario. ${ }^{37}$ Esto se confirma indirectamente en una publicación que encontró que para las portadoras de $B R C A 2$, el tener dos o más abortos terapéuticos se asoció con disminución del riesgo. ${ }^{39}$ En Islandia también se ha visto el efecto negativo del aumento de la paridad en estas mujeres. ${ }^{40}$ Sin embargo, un reciente estudio con una cohorte grande de portadoras de los genes de predisposición al cáncer mamario, BRCA1 y BRCA2, determinó que las portadoras de mutaciones en cualquiera de estos genes y mayores de 40 años, mostraron una reducción similar en el riesgo de cáncer de mama conforme aumentó la paridad, como sucede también en las mujeres de la población general. ${ }^{41}$ Otro estudio epidemiológico similar confirma estos datos y sugiere que el riesgo de las portadoras de mutaciones en $B R C A 1$ y $B R C A 2$ de desarrollar cáncer en relación con la paridad es el mismo que el de la población general, y que la historia reproductiva puede servir para mejorar las predicciones de riesgo en las portadoras. ${ }^{42}$ 


\section{Riesgo de heredar el cáncer a su prole ${ }^{3}$}

Este riesgo existe, pero solamente para los afectados por los raros síndromes de cáncer hereditario.

Si el síndrome se hereda de forma autosómica dominante, eso implica que el cáncer se manifiesta en una persona que tiene, en el par de cromosomas, la mutación en un solo cromosoma del par; el otro cromosoma funciona normalmente. Las características de este tipo de herencia son: a) el cáncer se transmite de generación en generación; b) casi siempre se ve en un solo lado de la familia, la paterna o la materna; c) tanto los varones como las mujeres pueden heredar y transmitir el cáncer a su prole; d) se puede ver la transmisión de hombre a hombre; e) la prole tiene un 50\% de riesgo de heredar la mutación y un $50 \%$ de probabilidad de heredar el cromosoma sano, y f) puede parecer que el cáncer se brinca una generación por varias razones, ya sea por penetrancia incompleta, muerte precoz por alguna otra causa, inicio del tumor en etapas tardías de la vida, o escasez de mujeres o de hombres en el pedigrí, cuando el órgano diana es específico de un sexo. Es posible que una persona tenga una mutación nueva, de manera que sea la primera persona afectada con cáncer de su familia, el cual puede transmitir de forma autosómica dominante a su descendencia. La mayoría de los síndromes de susceptibilidad al cáncer se heredan de esta manera (Cuadro 2). En el caso del síndrome de cáncer de mama/ovario, por ejemplo, las personas heredan una mutación germinal en $B R C A 1$ o $B R C A 2$; con solo que la copia de $B R C A$ que funciona normalmente adquiera una mutación somática en una célula mamaria/ovárica en algún momento de la vida, se pierde la función protectora que tenía esta copia y se puede desarrollar el cáncer. ${ }^{33}$

En el caso de herencia autosómica recesiva, la persona con cáncer tiene la mutación en los dos cromosomas del par, y tiene las siguientes características: a) los casos de cáncer se ven en una sola generación, generalmente no aparecen en generaciones sucesivas; $b$ ) los afectados suelen agruparse en una hermandad; c) los genes mutados vienen de ambos lados de la familia, materna y paterna; d) tanto el padre como la madre de la persona afectada le heredaron un cromosoma del par con la mutación; d) los padres tienen un cromosoma con la mutación y el otro miembro del par sano; e) como tienen un cromosoma sano, los padres no manifiestan la enfermedad o presentan solo algunos rasgos de esta, y f) el riesgo para esta pareja de tener más hijos afectados es del $25 \%$ para cada embarazo. Algunos síndromes de susceptibilidad al cáncer con este tipo de herencia son: el de Bloom, la ataxia telangiectasia y la anemia de Fanconi.

No siempre la historia familiar es fácil de interpretar; puede haber muchos otros factores que hacen difícil reconocer el patrón de herencia correcto en determinados pedigríes, tales como: familias pequeñas, muertes precoces, remoción quirúrgica de los órganos diana, falsa paternidad, falta de penetrancia, expresión variable, heterogeneidad genética, impronta genómica, mutaciones de novo, mosaicismo, herencia mitocondrial, consanguinidad, utilización de reproducción asistida, etc.

Para complicar el cuadro, cuando existe susceptibilidad hereditaria la probabilidad de enfermar de cáncer varía según el síndrome, la familia, el gen, las diferentes mutaciones en un mismo gen $\mathrm{y}$, posiblemente, otros factores aún desconocidos.

\section{Conclusiones}

Aunque la incidencia del cáncer aumenta conforme envejece la persona, un número importante de casos se presenta durante la infancia, la adolescencia y la edad reproductiva. Gracias a la constante mejora en metodologías para el diagnóstico precoz y para el tratamiento, la cantidad de sobrevivientes jóvenes es cada día mayor. A estas personas no basta con prolongarles la vida, es indispensable, además, garantizarles la buena calidad de esta. Un aspecto fundamental para muchas de ellas es conservar la capacidad de procrear. Una vez conseguido el embarazo, las perspectivas en cuanto a la salud del producto aparentemente son las mismas que para la población general. Las complicaciones obstétricas se presentan en algunos casos, en particular en las embarazadas sometidas a irradiación pélvica antes de la pubertad, y suelen relacionarse con restricción del crecimiento intrauterino fetal. El embarazo per se, cuando ocurre antes de dos años después del diagnóstico de la malignidad, podría ser perjudicial para algunas mujeres con antecedente de cáncer de mama y alto riesgo asociado. La posibilidad de heredar el cáncer a la criatura existe únicamente para un grupo reducido de portadoras de mutaciones asociadas con síndromes de cáncer hereditario.

\section{Abstract}

Survivors of pediatric, adolescent or pre-menopausal cancer also deserve good quality of life. An important aspect of personal roll being is related to procreation. It is possible that some of the therapeutic interventions which have saved their lives could have jeopardized their fertility, their future deliveries or their offspring health. Hereditary transmission of cancer is another source of concern. Cancer is a new paradigm for genetic counseling. All these issues are approached in this review. 


\section{Referencias}

1. Baker DL, Schuette JL, Uhlmann WR, editores. A guide to genetic counseling. New York: Wiley - Liss; 1998.

2. Harper PS. Practical genetic counseling. $5^{\text {th }}$ ed. Oxford: Butterworth - Heinemann; 1998

3. National Cancer Institute. Elements of cancer genetics risk assessment and counseling $\left(\mathrm{PDQ}{ }^{\circledR}\right)$. Health professional version. Last modified 01/18/2007. Disponible en: http://www.nci.nih.gov.

4. Robinson LL, Green DM, Hudson M, Meadows AT, Mertens AC, Packer RJ, et al. Long - term outcomes of adult survivors of childhood cancer. Cancer. 2005; 104: 2557-64.

5. Sklar CA, Mertens AC, Mitby P, Whitton J, Stoval M, Kasper C, et al. Premature menopause in survivors of childhood cancer: a report from the Childhood Cancer Survivor Study. J Natl Cancer Inst. 2006; 98 : 890-6.

6. Chemaitilly W, Mertens AC, Mitby P, Whitton J, Stoval M, Yasui Y, et al. Acute ovarian failure in the childhood cancer survivor study. J Clin Endocrinol Metab. 2006; 91: 1723-8.

7. Schover, LR. After the deluge, the emerging landscape of childbearing potential in pediatric cancer survivors. J Natl Cancer Inst. 2006; 98:1434-35

8. Brougham MF, Wallace WH. Subfertility in children and young people treated for solid and haematological malignancies. Br J Haematol. 2005; 131:143-55.

9. Lee SJ, Schover LR, Partridge AH, Patrizio P, Wallace WH, Hagerty $\mathrm{K}$, et al. American Society of Clinical Oncology recommendations on fertility preservation in cancer patients. J Clin Oncol. 2006; 24: 29172931.

10. Falcone T, Bedaiwy MA. Fertility preservation and pregnancy outcome after malignancy. Curr Opin Obstet Gynecol. 2005; 17:21-6.

11. Sonmezer M, Oktay K. Fertility preservation in female patients. Hum Reprod Update. 2004; 10:251-66.

12. Arnon J, Meirow D, Lewis-Roness H, Ornoy A. Genetic and teratogenic effects of cancer treatments on gametes and embryos. Hum Reprod Update. 2001; 7:394-403.

13. Byrne J, Rasmussen SA, Steinhorn SC, Conneally RR, Myers MH, Lynch CF, et al. Genetic disease in offspring of long-term survivors of childhood and adolescent cancer. Am J Hum Genet. 1998; 62:45-52

14. Meistrich ML, Byrne J. Genetic disease in offspring of long term survivors of childhood and adolescent cancer treated with potentially mutagenic therapies. Am J Hum Genet. 2002; 70:1069-71.

15. Boyce JD, Tawn E, Winther JF, Donaldson SS, Green DM, Mertens AC, et al. Genetic effects of radiotherapy for childhood cancer. Health Physics. 2003; 85: 65-80.

16. Tawn EJ, Whitehouse CA, Winther JF, Curwen GB, Rees GS, Stoval $\mathrm{M}$ et al. Chromosome analysis in childhood cancer survivors and their offspring - no evidence for radiotherapy - induced persistent genomic instability. Mutat Res. 2005; 583: 198-206.

17. Rees GS, Trikic MZ, Winther, JF, Tawn EJ, Stoval M, Olsen JH, et al. A pilot study examining germline minisatellite mutations in the offspring of Danish childhood and adolescent cancer survivors treated with radiotherapy. Int J Radiat Biol. 2006; 82: 153-60.

18. Meirow D, SchiffE. Appraisal of chemotherapy effects on reproductive outcome according to animal studies and clinical data. J Natl Cancer Inst Monogr. 2005; 34:21-5.

19. Critchley HO, Wallace WH. Impact of cancer treatment on uterine function. J Natl Cancer Inst Monogr. 2005; 64-8.

20. Signorello LB, Cohen SS, Bosetti C, Stovall M, Kasper CE, Weathers RE, et al. Female survivors of childhood cancer: preterm birth and low birth weight among their children. J Natl Cancer Inst. 2006; 98:1453-61.
21. Green DM, Whitton JA, Stovall M, Mertens AC, Donaldson SS, Ruymann FB. Pregnancy outcome of female survivors of childhood cancer: a report from the Childhood Cancer Survivor Study. Am J Obstet Gynecol. 2002; 187:1070-80.

22. Lantinga GM, Simons AH, Kamps WA, Potsma A. Inminent ovarian failure in childhood cancer survivors. Eur J Cancer. 2006; 42: 1415-20.

23. Van Dalen EC, van der Pal HJ, van den Bos C, Kok WE, Caron HN, Kremer LC. Clinical heart failure during pregnancy and delivery in a cohort of female childhood cancer survivors treated with anthracyclines. Eur J Cancer. 2006; 42: 2549-53.

24. Calhoun K, Hansen N. The effect of pregnancy on survival in women with a history of breast cancer. Breast Dis. 2005-2006; 23: 81-6.

25. Kroman N, Mouridsen HT. Prognostic influence of pregnancy before, around, and after diagnosis of breast cancer. Breast. 2003; 12: 516-21.

26. Park M, Davidson R, Fox K. Preservation of fertility and the impact of subsequent pregnancy in patients with premenopausal breast cancer. Semin Oncol. 2006, 33: 664-71.

27. Banks E, Reeves G. Pregnancy in women with a history of breast cancer. BMJ. 2007; 334:166-7.

28. Ives A, Saunders C, Bulsara M, Semmens J. Pregnancy after breast cancer: population based study. BMJ. 2007; 334:194-199.

29. Travis LB, Hill D, Dores GM, Gospodarowicz M, van Leeuwen FE, Holowaty E, et al. Cumulative absolute breast cancer risk for young women treated for Hodgkin lymphoma. J Nat Cancer Inst. 2005; 19:1428-37.

30. Hill DA, Gilbert E, Dores GM, Gospodarowicz M, van Leeuwen FE, Holowaty $\mathrm{E}$ et al. Breast cancer risk following radiotherapy for Hodgkin lymphoma: modification by other risk factors. Blood. 2005; 106: 3358-65.

31. Consejo Nacional de Cáncer. Normas y Procedimientos para el tratamiento de cáncer en Costa Rica. 2005.

32. Fenlay J, Bray F, Pisani P, Parkin DM. GLOBOCAN 2002: cancer incidence, mortality and prevalence. Lyon IARC Press, 2004. Disponible en: www-dep.iarc.fr/globocan/downloads.htm

33. Bennet RL, Hampel HL, Mandell JB, Marks JH. Genetic counselors: translating genomic science into clinical practice. J Clin Invest. 2003;112:1274-79.

34. Pharoah PD, Day NE, Duffy S, Easton DF, Ponder BA. Family history and the risk of breast cancer: a systematic review and meta-analysis. Int J Cancer. 1997; 71: 800-9.

35. Friedenson B. Assessing and managing breast cancer risk: clinical tools for advising patients. Med Gen Med. 2004; 6(1):8.

36. Risch HA, McLaughlin JR, Cole DE, Rosen B, Bradley L, Fan I, et al. Population BRCA1 and BRCA2 mutation frequencies and cancer penetrances: a kin-cohort study in Ontario, Canada. J Nat Cancer Inst. 2006; 98:1694-1706.

37. Narod SA. Modifiers of risk of hereditary breast cancer. Oncogene. 2006; 25.5832-6.

38. Cullinane CA, Lubinski J, Neuhausen SL, Ghadirian P, Lynch HT, Isaacs $\mathrm{C}$, et al. Effect of pregnancy as a risk factor for breast cancer in BRCA1/BRCA2 mutation carriers. Int J Cancer. 2005; 117:988-91.

39. Friedman E, Kotsopoulos J, Lubinski J, Lynch HT, Ghadirian P, Neuhausen SL, et al. Spontaneous and therapeutic abortions and the risk of breast cancer among BRCA mutation carriers. Breast Cancer Res. 2006; 8:R15

40. Tryggvadottir L, Olafsdottir EJ, Gudlaugsdottir S, Thorlacius S, Jonasson JG, Tulinius H, et al. BRCA2 mutation carriers, reproductive factors and breast cancer risk. Breast Cancer Res. 2003, 5:R121-R128 (DOI 10.1186/bcr619).

41. Andrieu N, Goldgar DE, Easton DF, Rookus M, Brohet R, Antoniou $\mathrm{AC}$, et al. Pregnancies, breast-feeding, and breast cancer risk in the International BRCA1/2 Carrier Cohort Study (IBCCS). J Natl Cancer Inst. 2006; 98:535-44.

42. Antoniou AC, Shenton A, Maher ER, Watson E, Woodward E, Lallo $\mathrm{F}$, et al. Parity and breast cancer risk among BRCA 1 and BRCA 2 mutation carriers. Breast Cancer Res. 2006; 8:R72. 